\title{
Der Ort der Bücher in der Bibliothek und im Katalog am Beispiel von Herzog Augusts Wolfenbütteler Büchersammlung
}

\section{Bücher-Ordnungen}

Mit jedem gedruckten und gehandelten Buch ist etwas gegeben, das die Absicht des Autors wie auch die Interessen des Verlegers übersteigt; etwas wird öffentlich. Schon das Drucken von Texten ist ein Akt der Konmmunikation, der Repräsentation, der Erwartung, der Bedürfnisbefriedigung, des Tauschens und Austauschens geistiger Gïter. Erst recht aber zeigt sich diese Dynamik in der Wirkung, die Bücher haben. Bücher sind wie Figuren auf der Bühne: Einmal in der Welt, behaupten sie ein eigenes Leben, das ganz wesentlich im Bezug auf andere Bücher besteht, reale oder fiktive, gelesene oder erträumte, anregende oder gefürchtete, so dass jeden Raum voller Bücher im Grunde ein vielstimmiges Konzert ordnender Stimmen erfüllen würde, könnte man die rhetorischen Verhältnisse zwischen ihnen hörbar machen: sie adressieren einander, kritisieren einander, spekulieren übereinander, schlieBen sich zusammen, grenzen sich ab usw.

Jedes Buch enthält explizite oder implizite Verweise auf andere Bücher. Ein Stich in Jacob Burckhards Geschichte der Wolfenbütteler Bibliothek von 1745 illustriert dies schlagend: "Liber librum aperit « - ein Buch öffnet ein anderes Buch (Abb. 1). ${ }^{1}$ Gewiss beziehen sich Bücher auf vieles, was nicht wiederum ein Buch ist - Meinungen, Annahmen, Thesen, Lebensverhältnisse, Sachverhalte, Erfahrungen und Gefühle - immer aber beziehen sie sich auch auf andere Bücher. Sie bilden, jedes für sich, eine Bibliothek, projizieren diese aus sich heraus: Sie dekretieren kanonische Werke und stiften so neue Zusammenhänge, sie verwerfen ganze Traditionen, die eben durch die Verwerfung als Segment der Bibliothek bestätigt werden, sie geben sich selber die Funktion eines Schlüssels für das Reich der Texte, sie organisieren Ordnungen, die auf sie selbst hin orientiert sind.

Diese Beziehungen sind schwierig und komplex, niemals einfach. Für einen Bibliothekar wird die bibliographische Vieldeutigkeit jedes Buches ein praktisches Problem seiner Aufstellung und Verzeichnung. Schon das dritte Buch, wenn es einer Sammlung von zweien hinzugefügt wird, hat mehrere mögliche Orte im Regal, um seine Beziehungen $z u$ den beiden anderen auszudrücken: vor den ersten, zwischen den beiden, nach dem zweiten. Ein Beispiel: Will man den Leviathan von Thomas Hobbes vor dieMeditationen des René Descartes stellen, weil sein Autor früher geboren wurde, oder zwischen die Meditationen und Baruch Spinozas Ethi$c a$, weil es dann nach Veröffentlichungsdatum konsekutiv eingeordnet wäre, oder gar nach diesem, weil etwa die englischen Bücher hinter die französischen und holländischen sortiert werden? Drei Kriterien der Ordnung, drei Möglichkeiten der Aufstellung, und es gibt noch viele andere: das Alphabet des Verfassernamens, die Größe des Buclıs, eine sachliche Gruppierung, eine Anordnung nach ideologischen oder politischen Kriterien, nach Anschaffungszeit usw. Jean Pauls Schulmeisterlein Maria Wutz, der die Bücher, die er lesen wollte, selber schrieb, wäre diesem Problem ebenso ausgeliefert gewesen wie jeder, der nur liest und nicht selber schreibt.

Sobald es Bücher gibt, werden ihre Ordnungen problematisch - nicht weil sie schwer auszumachen wären, sondern weil ihrer so viele Möglichkeiten existieren. Es nutzt gar nichts, von Büchern etwas $\mathrm{zu}$ verstehen, wenn man eine Bibliothek aufbauen will, im Gegenteil: Jedes Buch begehrt viele Plätze und kann doch nur einen haben, jedenfalls im Regal. Das ist das zentrale Problem der Bibliothek: Ordnung heißt für sie Verortung. Bücher werden im Regal abgestellt, und wo immer sie stehen, verletzt und beschränkt das die Ordnungen, die sie entwerfen, in die sie sich selbst entworfen haben, in denen sie entworfen werden. Die Frage WAS? wird in der Bibliothek gekoppelt an die Frage WO?, und diese

1 Jacob Burckhard: Historia Bibliothecae Augustae quae Wolffenbutteli est. 3 Bde. Leipzig: Typis Breitkopfianis 17441746 , Bd. 2 (1745), S. 145. 


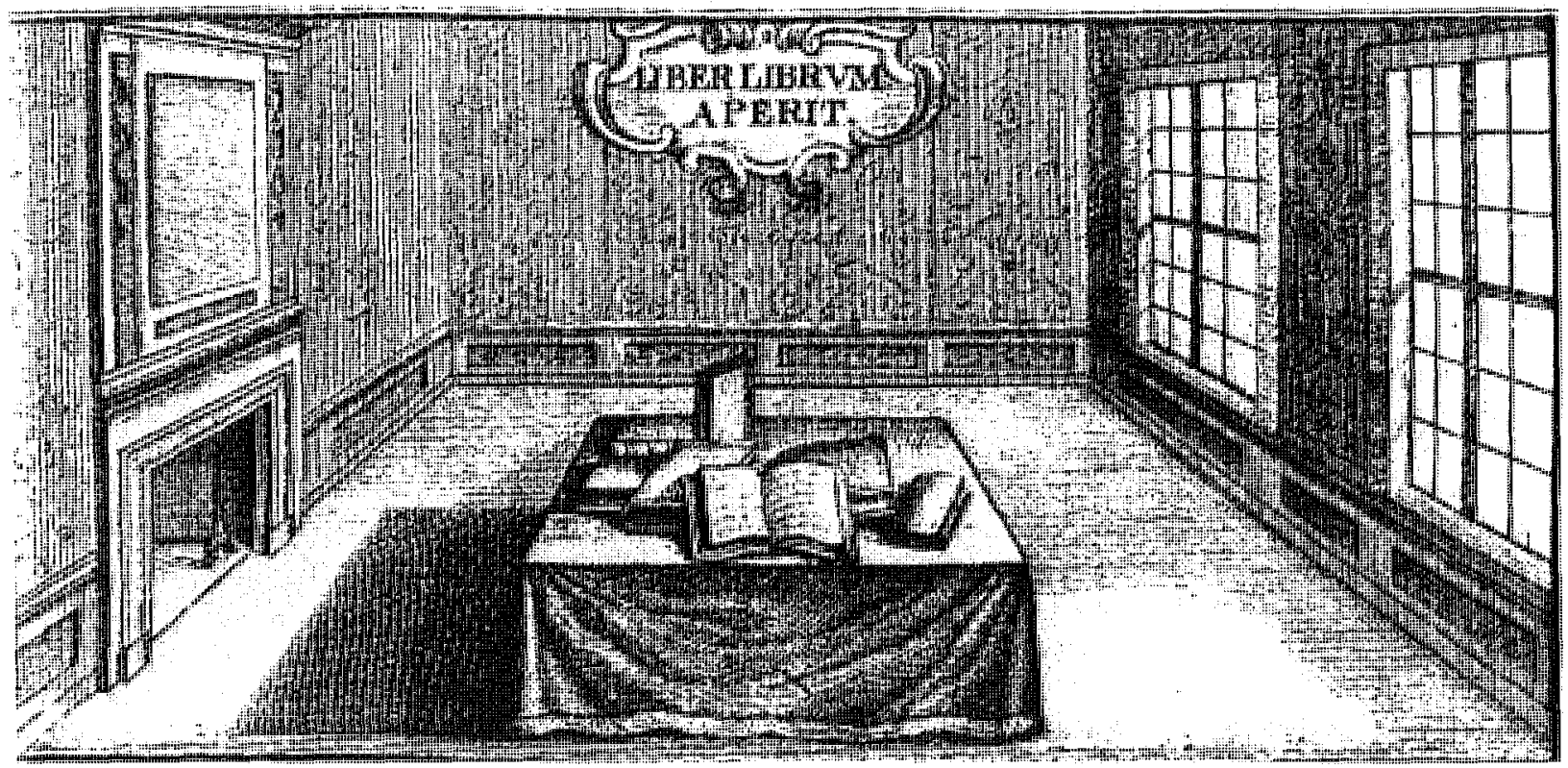

Abb. 1: Vignette aus der Bibliotheksgeschichte von Burckhard (1745).

Koppelung war und ist sowohl ein Problem der Erkenntnis wie eines der Praxis des Umgangs mit Büchern, der sich durch die Bibliothek und mit der Bibliothek realisiert.

\section{Katalog-Ordnungen}

Herzog August hat in seinen ersten Katalog, den er 1611 begann und 1625 beendete, einen lateinischen Sinnspruch von Erasmus von Rotterdam hineingeschrieben, der die Lust beschwört, im Wald von Büchern zu jagen, lieber als in der wirklichen Natur: "Nullis in saltubus, venatus jucundior, quam in $\mathrm{Bi}$ bliothecis «. ${ }^{2}$ Der Bibliothekar als Wanderer in Sinnwelten, Bedeutungswäldern und Wissensgestrüpp hat ein Vergnügen daran, diese zweite Natur zu ordnen, wenigstens einen Weg hindurch zu bahnen. Denn er weiß nur zu gut, wie es in einem Gedicht von 1654 heißt, das einen großen Bibliothekar und seine Sammlung preist:

Da kan ein iglich Buch ein Zeiger seyn/ dabey // Man mercken kan/was Recht/ was Lincks im Reisen sey.// Obschon ein Zeiger hie/ ein andrer dorthin weiset // So macht doch mit Bedacht das einer richtig reiset. ${ }^{3}$

Das poetische Bild war auf Herzog August (15791666) und seine Bibliothek gemünzt, die seit 1644 in Wolfenbüttel stand, nachdem sie zuvor ab 1604 in Hitzacker an der Elbe zielstrebig aufgebaut wurde. "Alles mir Bedacht", war die Devise des Fürsten, der als Bücherkäufer und -besitzer zugleich sein ei- gener Bibliothekar wurde und ganz offensichtlich in barocker Weise stolz auf seine Sammlung war. Die Bibliothek, die seinen Namen trägt, steht immer noch in Wolfenbüttel, wohin er sie überführte, nachdem er spät und einigermaßen unvorhergesehen Herzog von Braunschweig und Lüneburg geworden war. Zu dieser Sammlung gehörte auch ein Katalog, mit dem August die Kartographie seiner Bücherwelt realisierte - ein einmaliges Dokument seiner Arbeit. Bei allem Tempo des Bucherwerbs und bei allen Amtsgeschäften im letzten Drittel seines Lebens gab er es nie auf, im selbstgeschaffenen Wald der Texte eigene Wege zu bahnen, als Autor und als Leser. Sein erster Katalog (für die Jahre 1611-1625) füllt 360 Blätter eines kleinen Folioformats (Abb. 2).

Nach dem Alphabet der Autoren bzw. (bei Anonyma) der Titel in Gruppen eingeteilt, erhielten hier die Bücher innerbalb jeder der Buchstaben-Rubriken fortlaufende Nummern von 1 bis meist ca. 350. Diese Nummern scheinen nicht in die Bücher selbst eingetragen worden zu sein, was dafür

2 Als Nachweis unter diesen Zeilen wird Erasmus von Rotterdams Einleitung zu Lorenzo Vallas Kommentar zum Neuen Testament angegeben, vermutlich also: Laurentii Vallae: Viri Tam Graecae quam Latinae linguae doctissimi. In Novum Testamentum Annotationes cum Erasmi Praefatione. Basel: Lasins 1541, bei August die Signatur 1113 Theol. (1).

3 Joachim Lütkemann: Fautotum Clientum \& Ministrorum Vota et Mele, Wolfenbiuttel 1654. Zitiert nach: Alles mit Bedacht. Barockes Fürstenlob auf Herzog August (1579-1666) in Wort, Bild und Musik. Hrsg. von Martin Bircher und Thomas Bürger. Wolfenbüttel: Herzog August Bibliothek 1979, S. 161. 
spricht, dass es sich nicht um Buchsignaturen handelte. Die Blätter des Katalogs wurden einzeln beschriftet und erst später zusammengebunden. Ein Blatt, auf dem August einen Index specialis beginnen wollte, hat er für die normale Katalogisierung umgewidmet und die Index-Ankündigung durchgestrichen. Die sachliche Ordnung, wenn es eine gab, wurde nicht explizit gemacht oder gar nicht erst versucht.

1625 begann August seinen zweiten Katalog, der in über vier Jahrzehnten auf über 7.000 Folioseiten anwuchs, ein Dokument des Fleißes und der Sorgfalt, der Demut und der Neugier, das er bis 1648/49 eigenhändig produzierte. August schrieb 3.692 Seiten selbst, bevor ihn mit fast siebzig Jahren einerseits die Zeit dazu fehlte, andererseits seine Augen schlechter wurden, ${ }^{4}$ und er betreute persönlich die weitere Arbeit daran bis kurz vor seinem Tod. Dieses Dokument einer im 17. Jahrhundert neuen und bis heute einmaligen Anstrengung heißt , Katalog', aber dieser Begriff beschreibt schlecht, was hier eigentlich vorliegt: ein Dokument immensen Fleißes gewiss, aber auch ein Monument bibliothekarischer Vision, deren spezifische Lebendigkeit nur verstanden werden kann, wenn man sieht, was dieser sogenannte Katalog zur Sprache bringt.

Als Herzog August starb, verebbte auch die Katalogarbeit und damit das parallele Wachstum von Regalen und Verzeichnis, die dynamische Beziehung zwischen Sammlung und Verweisung. Erst im 20. Jahrhundert hat es in Wolfenbüttel eine staatliche Förderung ermöglicht, wieder »nach vorne zu kaufen«, wie das Lessing nannte, der im 18. Jahrhundert resigniert einräumen musste, dass sein bescheidenes Budget als Bibliothekar nur dazu reichte, »rückwärts" zu arbeiten: Lücken zu füllen und vielleicht Verweisungen zu ergänzen. Lessing be- tonte hundert Jahre nach August die Notwendigkeit eines neuen Katalogs für die nach des Herzogs Tode eingegangenen Bücher, und er meinte damals schon einen sachlichen oder Real-Katalog; ${ }^{5}$ dieser wurde jedoch erst in den für die Bibliothek noch ruhigeren Zeiten des 19. Jahrhunderts erstellt, als die Bibliothekare in Wolfenbüttel Zeit und Geduld zur Verzeichnung der sogenannten "nittleren Aufstellung * hatten. ${ }^{6}$ Die meisten Kataloge seitdem sind retro-

4 August unterzog sich Anfang der 1650er Jahre erfolgreich einer Star-Operation; vgl. Jill Bepler: Cultural Life at the Wolfenbïttel Court 1635-1666. In: A Treasure House of Books. The Library of Duke August of Brunswick-Wolfenbïttel. Wolfenbütel: Harrassowitz 1998, S. 131-146, bes. S. I40ff.

5 Bernd Reifenberg: Lessing und die Bibliothek. (Woltenbütteler Schriften zur Geschichte des Buchwesens. 23). Wolfenbüttel 1995 .

6 Paul Raabe: Die Herzog August Bibliothek Wolfenbùttel. Bestände - Kataloge - Erschließung. Wolfenbüttel: Heckners Verlag 1971 , S. $17-27,35 t$. 


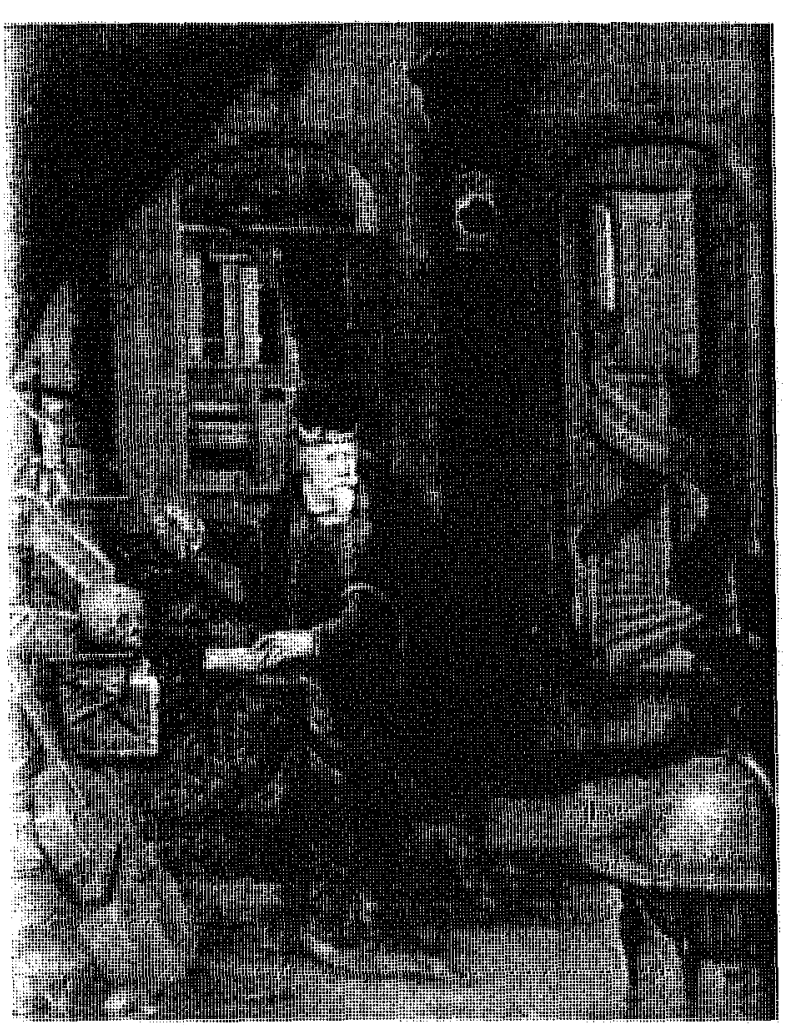

Abb. 3: Gemach des Herzogs (ca. 1659): Ort der Katalogisierung.

spektive Erschließungsleistungen - im Übrigen nicht nur in Wolfenbüttel - und damit etwas ganz anderes als der Katalog von August.

Viel gerühmt war dieser Katalog, der schon zu des Herzogs Lebzeiten nach seinem Stand- oder vielmehr Lageort auch $:$ Bücherradkataloge hieß. In einem um die Mitte des 17. Jahrhunderts nach England geschickten Brief wird mitgeteilt, die schwedische Königin habe eine Abschrift erbeten und sei abschlägig beschieden worden. ${ }^{7}$ Den Vorschlag des Helmstedter Professors und Freundes Hermann Conring, den Katalog drucken zu lassen, hat Herzog August nicht aufgegriffen. Der Katalog war geheim, er war verborgen, Attribut seines Autors, nicht Instrument irgendwelcher Leser. Ein Gemälde, das auf 1659 datiert wird und worauf der Herzog in der Tradition der Hieronymusdarstellungen in einem mit den Insignien der Gelehrsamkeit und der Christlichkeit voll gestopften Raum der einsamen Arbeit zu sehen ist, könnte - idealisiert - das Gemach darstellen, worin August am Katalog arbeitete (Abb. 3). Die dargestellten Bücher sind vielleicht Kataloge, aber das ist Spekulation. Das Bücherrad ist nicht zu sehen (Abb. 9). Darauf wären die Katalogbände jederzeit aufgeschlagen anzutreffen gewesen.
Über die Katalogwerkstatt Augusts gibt es keine Berichte oder Ansichten. Unter den Bildern der Bibliothek aus der Zeit des 17. Jahrhunderts ist der Katalog selbst nirgends auszumachen. Er war ein Schatz, eine Kostbarkeit, jedenfalls ein Geheimnis für die Zeitgenossen. Vielleicht dachten sie sich ihn als Schlüssel zur Sammlung, als `Sesam-öffne-dich` zum Tresor des angehäuften Wissens. Die Bibliothekare der unmittelbar folgenden Zeit fanden ihn hauptsächlich umständlich, unpraktisch, ärgerlich. Es half auch nichts, dass den sechs dicken Bänden mit jeweils über tausend Seiten noch zwei ebenso dicke Bände mit einem ab 1664 sauber neu geschriebenen alphabetischen Index der Verfassernamen als Hilfsmittel beigesellt wurden. So mühte sich der unter Gottfried Wilhelm Leibniz 1705 als Bibliothekar in Wolfenbüttel eingesetzte Lorenz Hertel mit der Suche nach einem bestimmten Werk von Cicero ab und klagte nach mehrmaligem Hin und Her zwischen alphabetischem Index und Hauptkatalog: "Warum nun diese Ordnung von dem Hochsel. Augusto beliebet worden, finde ich keine Nachricht. ${ }^{8}$ Eine Synthese der alten Instrumente musste her; sie wurde in fünfjähriger Arbeit während der Periode von Leibnizens Zuständigkeit für die Wolfenbütteler Sammlung produziert: ein Tirelkatalog mit alphabetischer Ordnung nach Verfassernamen (Abb. 4). ${ }^{9}$

Im ersten Jahrzehnt des 18. Jahrhunderts abgeschlossen, diente dieser $»$ Leibniz «-Katalog über 250 Jahre lang, bis zur Erstellung eines Zettelkatalogs mit Titelblattkopien im späten 20. Jahrhundert, als zentraler Zugang zur herzoglichen Samnlung. Für diejenigen, die um die partielle Unvollständigkeit dieses so genannten Titelblattkatalogs und folglich auch der daraus erstellten elektronischen Einträge im OPAC wissen, ist er der präferierte Zugang noch heute. ${ }^{10}$ (Leibniz soll versucht haben, die Zettel für die einzelnen Titel auch nach sachlichen Gesichts-

7 Der Brief aus den Hartlib Papers, University Library Sheffield, 31/23/40A-B, wird zitiert von Jill Bepler: Duke August and the Hartlib Circle. In: A Treasure House of Books, S. 165-172, S. 172.

8 Bericht von Lorenz Hertel an Herzog Ludwig Rudolph, 1731. Zitiert in: Elisabeth Wedderkopf: Entstehungsgeschichte und Einrichtung alter Kataloge der Herzog August-Bibliorhek Wolfenbüttel. (Prüfungsarbeit der Hamburger Bibliotheksschule). Maschinenschriftliche Arbeit. Hamburg 1951, S. 9.

9 Ähnliche alphabetische Titelkataloge wurden in Kiel 1736 und in Göttingen 1776 begonnen; vgl. Ladislaus Buzas: Deutsche Bibliotheksgeschichte der Neuzeit (1500-1800). (Elemente des Buch- und Bibliothekswesens. 2). Wiesbaden: Reichert 1976, S. 141.

$10 \mathrm{Da}$ August in Sarnmelbänden gelegentlich vollständige Schriften ohme Titeiblatt aufnahm und im Katalog vetzeichnete, ist die Liste aller seiner Texte länger als die der retrospektiven Katalogisierung nitrels Titelblattkopie. 
punkten zu ordiren, was aber nur als Idee zu einem Plan überliefert ist. ${ }^{11}$ )

\section{Signaturen- verwaltung}

Der von Herzog August 1625 angelegte und zur Hälfte selbst geschriebene Katalog ist kein Instrument, mit dem man $B \ddot{u}-$ cher finden kann. Weder mit dem Autornamen noch mit dem Werktitel kommt man darin weiter, auch nicht mit der zu Grunde gelegten sachlichen Ordnung. Nur im ersten Band des Hauptkatalogs trägt zunächst die sachliche Ordnung, denn August hatte seine Bücher entsprechend seiner 19 Rubriken getrennt aufgestellt und bildete diese Aufstellung auf den Seiten 1 bis $1531 \mathrm{ab}$, beginnend mit den Libri theologici und endend mit den Quodlibetici. ${ }^{12}$

Bereits auf dem Vorsatzblatt seines ersten Katalogs, auf dem auch der Sinnspruch über die Lust am Bücherwald notiert ist, finden sich 20 Rubriken skizziert. Einzelne Verbesserungen der Rubrikentitel (Astronomica statt Astrologica, Oeconomica statt Rustica etc.) und Umsortierungen in der Reihenfolge (Poetica wird nach oben verschoben) datieren wahrscheinlich aus der letzten Phase in den zwanziger Jahren des 17. Jahrhunderts, als die Fülle der Bücher - es waren damals schon über $6.000^{13}$ - nach Ordnung und Neugruppierung verlangten. Es sind diese skizzierten Rubriken jedenfalls identisch mit denen ganz vorne im 1625 begonnenen zweiten Katalog aufgeführten und bis zum Ende des 17. Jahrhunderts in Wolfenbüttel ver-

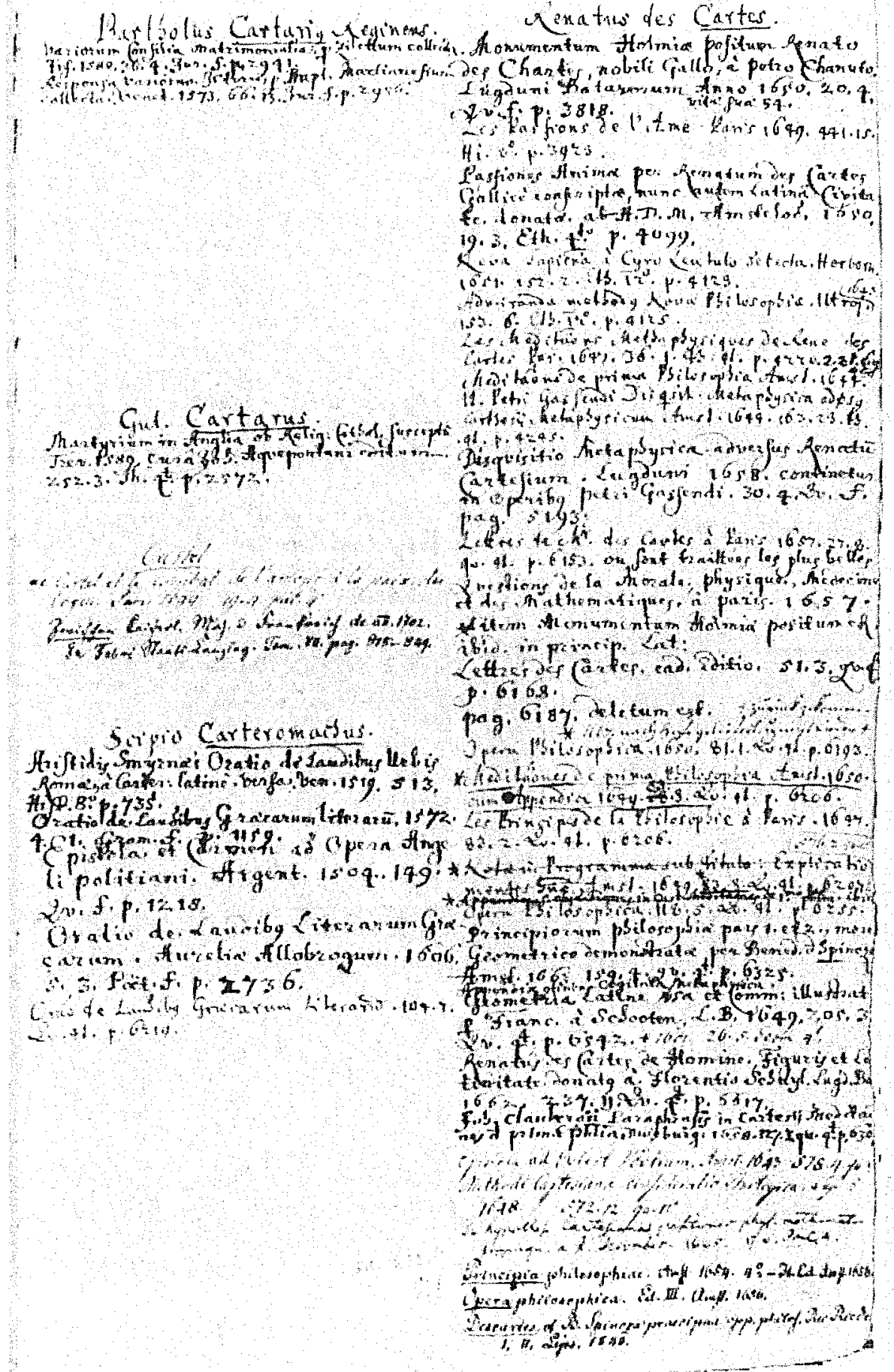

Abb. 4: Leibniz-Katalog (18. Jh.): Eintragung René Descartes.

wendeten Rubriken (mit der Ausnahme der Rubrik Dialectica, die in Logica umbenannt wurde): Theo-

11 Das sog. Numeralregister zum alphabetischen Karalog sei von Leibniz nicht vervollständigt worden, obwohl er seine Zettel systematisch ordnen wollte; vgl. E. Wedderkopf: Entstehungsgeschichte und Einrichtung alter Kataloge der Herzog August-Bib- 


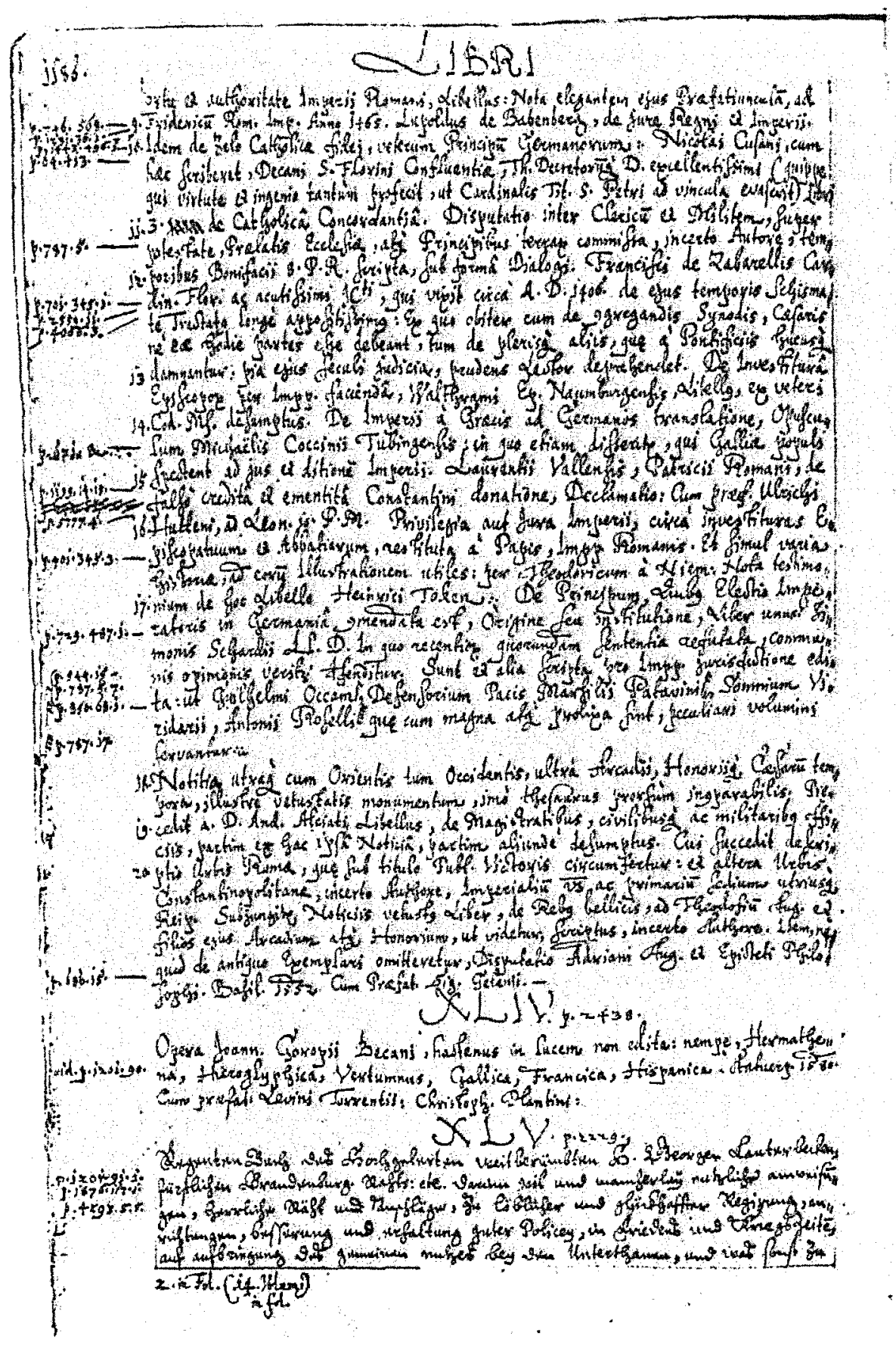

Abb. 5a: Bücherrad-Katalog, Seite 1186 (1627). Libri quodlibetici in folio 4345, Augusts Hand. Dieser frühe Eintrag zeigt die Aufnabme der bereits aufgestellten Sammlung; es werden ganze Zahlen in aufsteigender Reihe vergeben.

Größe, wobei die Folianten eine eigene Zählung erhielten. So erhielt vom größten Folianten mit der Nummer 1 der Größe nach abwärts jeder Band eine Nummer $1+\mathbf{n}$; was sich sodann einsetzend mit dem größten Quartband, der ebenfalls eine Nummer 1 erhielt, der Größe nach abwärts wiederholte: Auch hier erhielt jeder neue Band eine Nummer $1+n$, wobei Oktav- und Duodezbände numerisch einbegriffen, also durchgezählt wurden. ${ }^{14}$ In jeder Rubrik ergab sich eine kontinuierliche Reihe immer kleiner werdender Bände mit immer größer werdenden Zahlen. ${ }^{15}$ Manchmal wurden die Übergänge der Formate nahezu unsichtbar, bei großen Rubriken sogar der Übergang vom kleinsten Folioband zum größten Quartband. Augusts Band-Signaturen wurden auf dem Rücken der Bücher zugleich mit der Titelangabe eingetragen, bei Sammelbänden standen womöglich alle Titel aller Schriften darauf - ein regelrechtes Manuskript auf Pergament, das für den Besucher vor Ort den Inhalt der Regale entziffern half und welches das Manuskript des Katalogs im herzoglichen Gemach verdoppelte.

Dieser Katalog war anfangs ein Bestandsver-

logica, Juridica, Historica, Bellica, Politica, Oeconomica, Ethica, Medica, Geographica, Astronomica, Musica, Physica, Geometrica, Arithmetica, Poetica, Logica, Rhetorica, Grammatica, Quodlibetica, Manuscripta.

Die Bücher wurden aufgeführt, wie sie im Regal standen, also innerhalb der Rubriken strikt nach zeichnis, und für eine gewisse kurze Zeit entspra-

liothek Wolfenbüittel, S. 14: "Er [d. i. Leibniz] ließ von dem damaligen Sekretär der Wolfenbuitteler Bibliothek Sieverds sämtliche Titel der vorhandenen Bücher auf Bogen schreiben, und zwar so angeordnet, daiß auf jedem Bogen 32 Titel gleichmäßig verteilt waren. Die Bogen wurden nur einseitig beschrieben, geordnet. In einem weiteren Arbeitsgang sollte dann dieses Material zu dem 
chen die Stellen der Bücher im Regal den entsprechenden Signaturen im Katalog. Diese Arbeit war 1627 beendet und endete etwa bei den Theologica mit der Nummer 520 Folio bzw. 1340 in den anderen Formaten (zuletzt $12^{\circ}$ ), bei den Quodlibetica mit der Nummer 164 Folio bzw. 608 in den anderen Formaten (Abb. 5a). Nach Erledigung der Bestandsaufnahme wandelte sich der Katalog in der Hauptsache zum Akzessionsverzeichnis, demn nun trug August nacheinander diejenigen Werke ein, die er aus den Anlieferungen zusammenstellte bzw. übernahm (Abb. 5b).

Man vergesse nicht, dass August die Bücher meist eigens binden ließ eine platz- und kostensparende Maßnahme, die zugleich die Herstellung dickerer Buchblöcke erlaubte, deren Buchrücken sich wiederum besser zum Beschriften eigneten. ${ }^{16}$ Was im Regal zusammengebunden stand, wurde im Katalog ausführlich einzeln bibliographiert - August hat penibel und fast durchweg fehlerfrei den Titel reproduziert. Auch und gerade bei Sammelbänden war er sorgfältig: Es erhielt die jeweils zweite Schrift, als erste beigebundene Schrift, im Katalog unterhalb der Signatur die Nummer 1 usw. ${ }^{17}$

Das Akzessionsverzeichnis machte ein munteres Rubrikenspringen unvermeidlich, da die Bücher mehr oder weniger nach Eingang katalogisiert wurden. Neben die mittig gestellte Signatur, die jeden Eintrag eröffnete, schrieb August ein "vide" (man sehe) mit der Angabe derjenigen Katalogseite, auf

\section{VARIII}

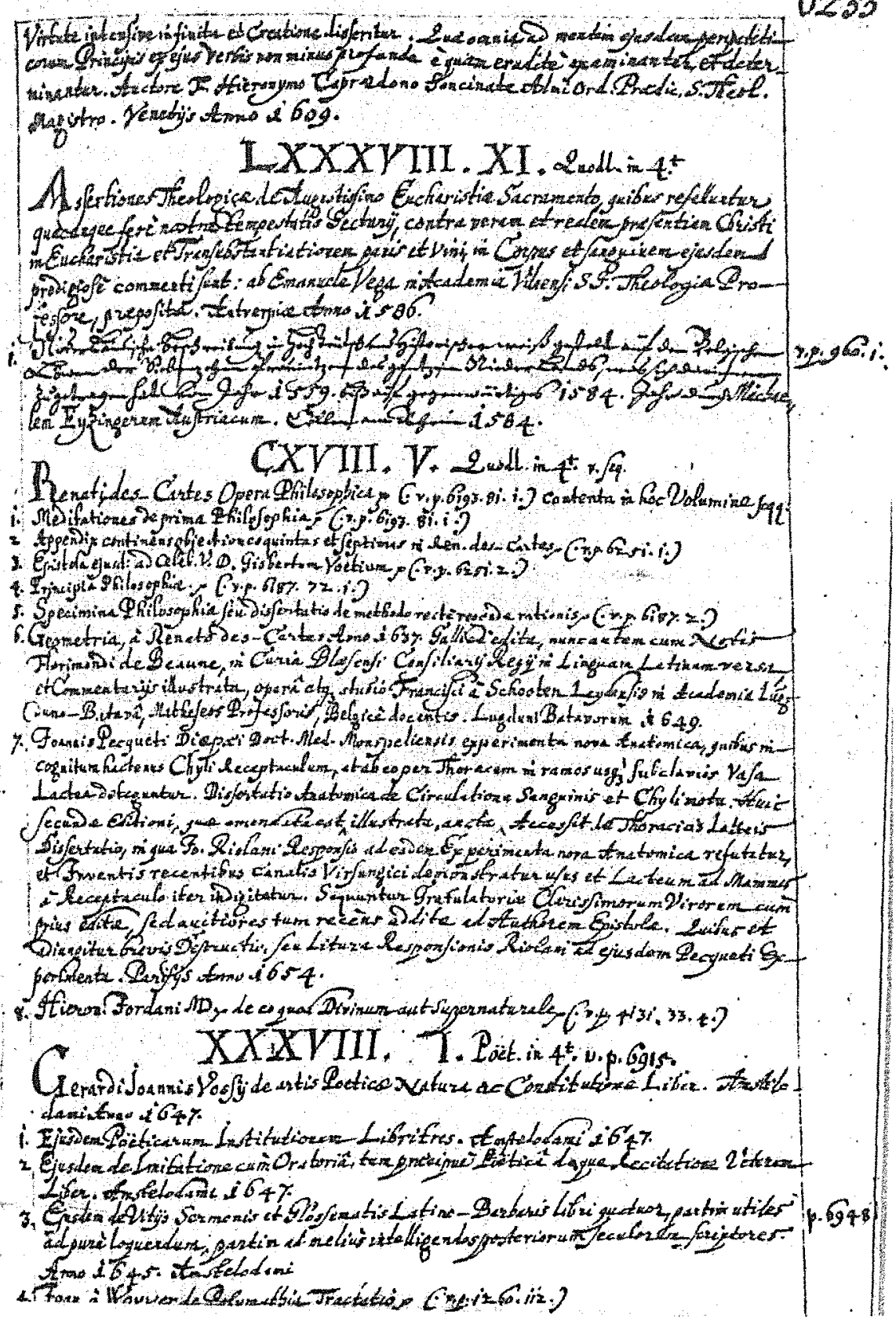

675

Abb. 5b: Bücherrad-Katalog, Seite 6255 (1667). Libri Varii, Hand von "Cancelist" Johann Heinrich Arlt. Dieser späte Eintrag zeigt das Aquisitionsverzeichnis mit springenden sachlichen Rubriken und Punkt-Signaturen für eingeschobene Werke. Die Signaturen werden mit Rubrik und Format angegeben. gewünschren alphabetischen Bandkatalog abgeschrieben werden. [Es folgt eine Fußnote mit dem Hinweis, daß »dieser Katalog von Leibniz angelegt wurde", daß also nicht der von Herzog August bereits angelegte benutzt wurde!] Anschließsend wollte Leibniz die Zettel daun in einer neuen Ordnung zu einem 'Catalogus Materiarum verwenden." 
der man die Fortsetzung der rubrikeninternen Zählung finden könne, also den nächsten Eintrag derselben Rubrik. Da auf jeder Seite oft mehrere Rubriken erscheinen, trug der Katalog durchgängig, also von S. 1531 bis zum Schluss auf S. 7200, die Kolumnentitel "Libri Varii «.

Der Katalog legte die Signatur fest, und das bedeutete, dass es je nach Format 19 Möglichkeiten der Einsortierung einer Druckschrift gab, wenn man von den 20 Rubriken die Manuscripta abzieht: 19 Folio-Stellplätze und 19 Stellplätze für die anderen Formate insgesamt. Welchen genauen Ort im Regal das Buch erhielt, entschied einzig und allein seine Größe, denn der Herzog sparte vor allem dadurch an Platz, dass er fixe Regalhöhen füllte. Die Sammlung ist heute noch so aufgestellt. Die Signaturenfolge in ganzen Zahlen wurde durch Neuzugänge notwendigerweise unterbrochen - weswegen der Herzog einen Punkt machte. Dieser untergliederte die Reihenfolge beliebig. Sollte etwa ein Band der Signatur Poetica 38.1 (s. Abb. 5b) nachgeordnet werden, so konnte er die Nummer 38.1.1 oder auch 38.2 erhalten (was in diesem Fall sogar beides geschah: der Verweis geht auf S. 6915).

Zur Kontrolle der bereits vergebenen Nummern wurde eine Liste bereits vergebener Signaturen geführt. Auf diese Weise sicherte das Signaturensystem eine eindeutige Beziehung zwischen Katalogeintrag und Stellplatz des Buches. Es machte allerdings zugleich diesen Stellplatz beweglich und den Katalogeintrag verweisungsbedürftig. Die Bibliothek war permanent in Bewegung, da neue Bände nicht hinten angefügt, sondern dazwischen geschoben wurden, was eine Unräumarbeit größeren Ausmaßes erforderlich machte. Es ist diese Dynamik, welche die Bibliothek Herzog Augusts auszeichnete, ihr rasches Wachstum von Null auf 135.400 Titel (Druckschriften und Manuskripte im Jahr 1666), repräsentiert in 31.300 Bänden.

Das Außergewöhnliche dieser Sammlung ist nicht nur die schiere Größe. Nach allen Zeugnissen ist mehr als klar, dass es August zunächst gar nicht auf Menge ankam: Der Herzog wählte fleißig aus und enttäuschte seine Agenten nicht selten, weil er Bücher zurücksandte, die diese auf eigene Rechnung besorgt und geschickt hatten. ${ }^{18}$ Es ist auch die Sorgfalt der Katalogisierung dieser Bibliothek, die man als Kriterium in Anschlag bringen muss.

\section{Ordnung und Verortung}

Es war die Dynamik des Wachstums, welche die Bildung größerer Sinneinheiten nicht zuließ und alles zerstreute: Autoren, Werke, Themen. Die herzogli- che Bibliothek ist, aus der Perspektive eines Zeitgenossen gesehen, eine komplexe und verrätselte Figur. So leicht es war, an den Regalen die einzelnen Werke an der Rücken-Information zu identifizieren, so schwer war es, ihre Ordnung zu durchschauen. Aus mehreren Faktoren entstand ein Monument, dessen Größe umgekehrt proportional zu seiner Einsehbarkeit war. Auch wenn man einige Interessen und Zielsetzungen benennen kann, ergibt sich noch lange kein einheitlicher Sinn. Sicher war der Herzog als Gelehrter ein humanistisch inspirierter Bücherkenner, als Lutheraner ein tief an Theologie Interessierter, ${ }^{19}$ und sicher waren seine Agenten intelligente Berater und fleißige Lieferanten. Auch war genügend Geld vorhanden, in allen Phasen von Augusts Leben: als Privatgelehrter in Hitzacker von 1604 bis 1634, danach als Regent in Braunschweig und ab 1643 in Wolfenbüttel. Dauer und Intensität des Monuments, welches die Bibliotheca Augusta und ihr Katalog darstellen, sind dennoch erstaunlich und völlig ohne Beispiel, und insofern nicht allein aus Motiven zu erklären. Wie viele andere adelige Büchersammler hatte August seine Bücher verliehen und wohl auch als Investition in Bildung ver-

12 Genauere Angaben bei Maria von Katte: Herzog August und die Kataloge seiner Bibliothek. In: Wolfenbütteler Beiträge 1 (1972), S. 168-199, S. 176f.

13 Katte: Herzog August und die Kataloge seiner Bibliothek, S. 171, nennt die genaue Zahl: 6.245. Dieser Aufsatz ist grundlegend für die hier vorgebrachten Ausführungen.

14 Auch hier gibt es Abweichungen: So wurden Bände, die größer als die Nr. 1 einer Reihe waren, mit Buchstaben bezeichnet, s. Katte, S. 192; ein Beispiel: B1 Arithm. $4^{\circ}$.

15 Auch wenn die Aufstellung nach Größe im 17. Jahrhundert ineist gängig war (Buzas: Deutsche Bibliotheksgeschichte der Neuzeit, S. 139f.), war eine entsprechende Signaturenvergabe die Ausnahme.

16 Vgl. Jochen Bepler: Zur Erinnerung unserer Hinfälligkeit. Zur Typologie des Sammlers Ferdinand Albrecht von Braunschweig Lüneburg. In: Jill Bepler (Hrsg.): Barocke Sammellust: die Bibliothek und Kunstkammer des Herzogs Ferdinand Albrecht zu Braunschweig-Lüneburg (1636-1687). Ausscellungskatalog. Weinheim: VCH, Acta humanoria 1988, S. 1323; über die Signaturen Augusts und die Aufstellung seiner Bibliothek S. 16.

17 Beim Titelblattkatalog des 20. Jahrhunderts und entsprechend beim elektronischen Katalog wurde aus der 1 eine 2, und jede folgende Nummer wurde ebenfalls um eine Zahl erhöht, um die Angabe "0 « zu vermeiden.

18 Einen guten Einblick in diese Praxis gibt Helnnar Härtel: Herzog August und sein Bücheragent Johann Georg Anckel. Studien zum Erwerbungsvorgang. In: Wolfenbütteler Beiträge 3 (1978), S. 235-282.

19 Vgl. Jörg Jochen Berns: Herzog August - Frömmigkeit und kirchliche Tradition. In: Sammler - Fürst - Gelehrter. Herzog August zu Braunschweig und Lüneburg 1579-1666. Katalog der Niedersächsischen Landesausstellung in Wolfenbüttel 1979, S. $343-378$, bes. S. 350 f. 
standen. ${ }^{20}$ Gleichzeitig rivalisierte er mit seiner eigenen Universität in Helmstedt und den dortigen Gelehrtenbibliotheken. Im Unterschied zu allen diesen ,Konkurrenten r aber besaß August einen genauen Katalog seiner Sammlung. Dieser Katalog macht den Eindruck eines abgeschlossenen, in sich geschlossenen Werks. Er ist aber im Gegenteil weit eher Textzeuge einer beinahe unendlichen Zersplitterung von Sinn, eben jener Zerstreuung rezipierbarer Einheiten wie Autor, Werk, Thema, die im schnellen Wachstum der Bibliothek nicht eingegrenzt, sondern sozusagen aufgefächert wurden.

Nehmen wir als Beispiel die Schriften des französischen Philosophen René Descartes (1596-1650), von dem in der herzoglichen Sammlung eine große Zahl an Erstausgaben vorhanden ist, wie in der Bibliographie von Matthijs van Otegem aufgeführt. ${ }^{21}$ Wir entnehmen dieser Bibliographie als erstes das Faktum, dass die Werke von Descartes in den Bibliotheken Frankreichs und Hollands, deren Bestände autoptisch überprüft wurden, fast immer als einzeln gebundene Bücher auftauchen, während bei August mit einer Ausnahme die Schriften des Franzosen zusammengebunden mit Scluriften anderer Autoren aufgestellt wurden. Sogar zwei Werkausgaben von Descartes hat der Herzog noch mit Schriften anderer Autoren zu großen Quartbänden vereinigt (Quod. 81.1 und 118.5). Der Autor 'Descartes ist damit eine Größe, die im Regal wie im Katalog nur in voneinander isolierten Elementen existiert und nur im alphabetischen Index als solche anzutreffen ist, wo wiederum die Werke nicht erkennbar sind. Der alphabetische Index verweist vom Namen nur auf die Seitenzahlen des Hauptkatalogs (Abb. 6).

Der neben dem Hauptkatalog von Anfang an geführte alphabetische Index schwoll unvermeidlich an solchen Stellen an, wo von einzelnen Autoren immer mehr Bücher verzeichnet wurden. Er musste darum überarbeitet werden, was bei August zum letzten Mal im Jahr 1664 begonnen wurde. Wo zuvor der permanente Zukauf die handschriftlichen Wucherungen veranlasste, die tendenziell unlesbar wurden, ${ }^{22}$ übernahm nun eine penible Registratur von Belegstellen die Verweisung.

Es zwang diese Indizierung bei der Suche nach einzelnen Werken, den Hauptkatalog immerzu aufzuschlagen und umzublättern. Das eben war es, was Lorenz Hertel zur Verzweiflung trieb und Leibniz am Anfang des 18. Jahrhunderts zur Abhilfe motivierte. Denn ohne Angabe von Werken und Signaturen war der alphabetische Index wie eine Chiffrierung - nicht der Bände in den Regalen, sondern allein ihres Nachweises im Katalog.

Der Ort eines Buches in der Bibliothek - wo ist er zu finden? Die Bücher sagen: Im Regal - lesen Sie

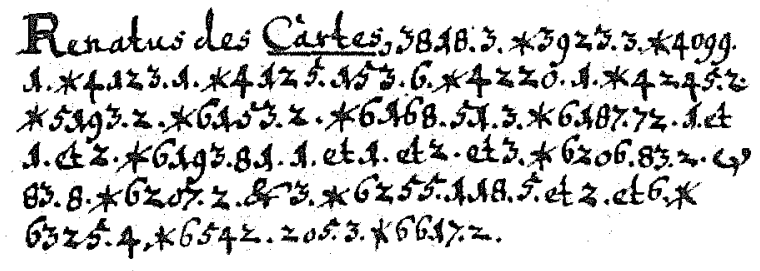

Abb. 6: Eintrag Descartes im alphabctischen Index ca. 1664 (Detail).

unsere Rücken! Der alphabetische Index sagt: Im Katalog, schlagen Sie die folgenden Seiten nach! Der Katalog selbst sagt: Blättern Sie mich durch, orientieren Sie sich an meinen internen Verweisungen hinter der Signatur, dann werden Sie alle sachlichen Rubriken virtuell durchlaufen. Mit anderen Worten: Vom Katalog führt kein gerader Weg ins Regal. Demn die Rubriken waren, fast ebenso wenig wie Autorname oder Werktitel, eine sachliche Ordnung, von der her man Bücher thematisch einkreisen konnte. Der Katalog ist eben auch kein sachliches Werkzeug der Identifizierung, im Gegenteil: Er ist zunächst und vor allem das Protokoll der Tatsache, dass Bücher durch die Hände des Herzogs gelaufen sind. Der Katalog ist eine herzogliche Checkliste, eine Membran für die aus dem Markt in die Regale beschleunigten Bücher. Endstation Wolfenbüttel, das hieß für die erworbenen Texte notwendig: Eingangskontrolle beim Katalogisierer, Aufnahme in eine repräsentative Wissensarchitektur. Aber es hieß nicht: sichere Verortung in den Rubriken, und also nicht: Ordnung durch Zuordnung.

Stellen wir uns vor, wir seien ein bedeutender Text, ein französischer Traktat vielleicht, den das allgemeine wissenschaftliche Interesse so weit auf den Wassern der öffentlichen Wahrnehmung nach oben getrieben hat, dass ein Agent des Herzogs auf uns aufmerksan wurde. Wir haben beispielsweise

$20 \mathrm{Vgl}$. Johannes Tütken: Höhere und mittlere Schulen des Herzogtums Braunschweig-Wolfenbütrel, der Herrschaft Dannenberg und der Grafschaft Blankenburg im Spiegel der Visitationsprotokolle des Generalschulinspektors Christoph Schrader (1650-1666). Wiesbaden: Harrassowitz 1997; Jean-Luc Le Cam: La politique scolaire d'Auguste le Jeune de BrunswickWolfenbüttel et l'inspecteur Christoph Schrader 1635-1666/80. 2 Bde. Wiesbaden: Harrassowitz 1996.

$21 \mathrm{Vgl}$. Matthijs van Otegem: A Bibliography of the Works of Descartes. 2 Bde. Utrecht: Zeno, The Leiden-Utrecht Research Institute of Philosophy 2002.

22 Vom ersten alphabetischen Index ist nur ein Band, erstellt 1627-1631, erhalten (HAB: BA I, 320). Der ab 1664 neugeschriebene Index wurde bis ca. 1770 erstellt, wobei aucl danach noch (bis 1693) Einträge gemacht wurden; vgl. Katte: Herzog August und die Kataloge seiner Bibliothek, S. $195 f$. 


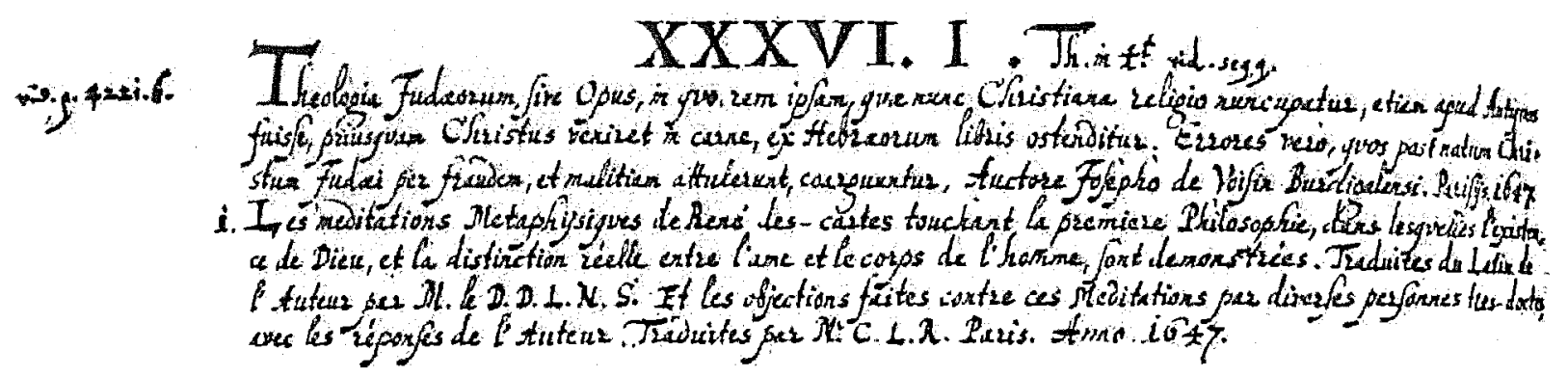

Abb. 7: Bücherradkatalog S. 4770 mit Titel »Méditations» von Descartes (Detail).

die Presse des Pariser Druckers Witwe von Jean Camusat und Pierre Petit« im Jahre 1647 verlassen und heißen - laut Titelblatt - Les méditations métaphysiques von René Descartes. Stolzes Produkt eines Autors, der durch Georg Wilhelm Friedrich Hegel im 19. Jahrhundert zum »Vater der modernen Philosophie « erklärt werden wird, ${ }^{23}$ sind wir durch den Agenten Abraham Wicquefort ${ }^{24}$ auf den Weg nach Wolfenbüttel geschickt worden und wiewohl Herzog August unseren späteren Ruhm noch nicht ahnen kann, finden wir Aufnahme in die Sammlung. Hier harren wir unseres späteren Schicksals, das neben Ruhm auch Verunglimpfung bringen wird, so wenn Martin Heidegger im 20. Jahrhundert das rationalistische Denken brandmarken und uns dafür als Beispiel nehmen wird. ${ }^{25}$ Noch nichts davon im 17. Jahrhundert: Herr Wicquefort wird bezahlt, wir werden gebunden und mit einer Signatur beschriftet, die uns unter die Theologica einreiht - dort streng nach Größe, versteht sich. Aber was besagt diese Einordnung?

Auf dem Weg ins Regal haben wir den Katalog passiert, der uns die Ehre einer recht genauen Aufnahme angedeihen lässt (Abb. 7) - wie üblich bei August, der "alles mit Bedacht" erledigen wollte und so stehen wir nun unter den Theologica als Nummer 36.1, zusammengebunden mit einem Buch über jüdische Theologie aus dem gleichen Jahr 1647. In einem zweiten Exemplar, das vermutlich später angeschafft wurde, stehen wir übrigens unter den Logica als Nummer 2.2.1 zwischen der Logica tripartita von Francisco González aus dem Jahre 1639 (Log. 2.1) und jesuitischen AristotelesKommentaren aus Coimbra, die 1607 erschienen (Log. 2.2); beide wurden eigens für uns auseinandergeschoben. Wir haben damit einen Platz nicht nur im Regal, sondern auch im Katalog, auf dessen entsprechende Seite eine Verweisung im alphabetischen Index referiert. Angekommen? Beinahe. Im Regal bleibt es ja unruhig, weil der Herzog die Anorduung nach Größe so streng befolgt, dass er immer wieder die Bücherreihen aufsprengt und durch Einschub verlängert. Das mache unseren Platz zwar relativ sicher, aber eben nur relativ: immer bezogen auf Nachbarn, deren Hutschnur sozusagen der unseren gleicht, aber keineswegs sicher im Sinn einer Feststellung unseres Themas. ${ }^{26}$

Wenn es den alphabetischen Index nicht gäbe, müssten wir unsere Buchgeschwister unglaublich lange suchen. So können wir wenigstens über Umwege erfahren, dass die meisten Schriften des Cartesius unter die Quodlibetica gerieten, weil sie mit anderen Texten anderer Autoren und anderer Wissenschaften zusammengebunden wurden. Das betrifft ältere Schriften wie den Discours de la méthode von 1637 (Quod. 64.25 (1)) oder die Prinzipien der Philosophie in einer Ausgabe von 1647 (Quod. 83.2 (1)), ebenso wie jüngere, nach uns angekaufte, wie die Geometria von 1649 (Quod. 205.3 (1)), die Schrift Über den Menschen in einer Ausgabe von 1662 (Quod. 237.11 (3)), sowie eine Briefedition von 1657 (Quod. 27.8 (3) und Quod. 51.3 (1)). Es betrifft auch die Texte der 1650 in Amsterdam erschienenen Opera Philosophica, die sich den Buchdeckel mit zwei Schriften über Anatomie (von Johannes Pecquetus) und über das Göttliche im sterblichen Körper (von Hieronymus Jordanus) teilen müssen und deshalb weit weg von den Logica in die ungleich größere Rubrik der Quodlibetica gerückt wurden (Quod. 118.5 (2)).

Dass wir ebenfalls unter den Quodlibetica ein Descartes nur zugeschriebenes, angeblich auf Be-

23 Hans-Peter Schürt: Die Adoption des "Vaters der modernen Philosophier. Studien zu einem Gemeinplatz der Ideengeschichte. Frankfurt am Main: Klostermann 1998.

$24 \mathrm{Vgl}$. Robert Mandrou: Abraham de Wicquefort et le duc August (1646-1653). Sur les relations intellectuelles entre France et Allemagne, un siècle avant les Lumières. In: Wolfenbiitteler Beiträge 3 (1978), S. 191-233.

25 Martin Heidegger: Die Zeit des Weltbildes [1937]. In: Martin Heidegger: Holzwege. Frankfurt am Main: Klostermann 1950, S. 69-104.

$26 \mathrm{Vgl}$. Ulrich Johannes Schneider: Bücher und Bewegung in der Bibliothek yon Herzog August. In: Sammein, Ordnen, Veranschaulichen: zur Wissenskompilatorik in der Frühen Neuzeit. Hrsg. von Frank Büttner, Markus Friedrich, Helmut Zedelmaier. Münster: Lit Verlag 2003, S. 111-127. 
fehl der schwedischen Königin Christine verfasstes Ballett von 1649 mit dem Titel Die Geburt des Friedens (Quod. $20.42^{\circ}(6)$ ) finden, muss uns nicht wundern; eher schon die Tatsache, dass Descartes' Brief an Voetium von 1644 nicht nur dort (Quod. 578.4), sondern in einer anderen Ausgabe auch bei den Poetica zu finden ist (Poet. 196.28 (5)), oder dass die Passiones Animae von 1650 sowohl in der Gesamtausgabe und damit bei den Quodlibetica (Quod. 81.1 (4)), aber auch unter den Ethica (Eth. 19.3 (3)) und unter den Historica (Hist. 441.15) stehen. Unverständlich aber muss uns erscheinen, dass die lateinischen Ausgaben unseres eigenen Textes der Meditationes einmal in einer Ausgabe von 1644 unter die Theologica (Theol. 163.23 (2)) eingereiht wurden und zum anderen in einer Ausgabe von 1650 unter die Quodlibetica (Quod. 81.1 (1)). ${ }^{27}$ Was ist das für ein System?

Ein System als sachlich strenge Ordnung gibt es hier nicht. August hatte zwar 19 Rubriken konzipiert, vermutlich kurz vor 1625 , dem Beginn der Arbeit am Hauptkatalog. Und er hat 1636 diese Rubriken auch zusammen mit der Bibliotheksordnung in der Bibliothek selber angeschlagen. Aber er hat damit weder ein topisches System, wie es Konrad Gessner im 16. Jahrhundert entwarf, noch ein Sachkatalogsystem, wie es im 18. Jahrhundert gängig wurde, etabliert. ${ }^{28}$ Es war keine Ordnung, die irgendetwas zusammenführte, sondern sozusagen eine Ordnung, die versuchsweise nicht alles zusammenstehen ließ.

In Augusts Bibliothek wurden die Werke des Descartes in die Rubriken Ethica, Logica, Theologica und Quodlibetica verteilt, letztere am häufigsten aus dem Grund des Zusammenbindens mit anderen Texten. Quodlibetica war der Titel Augusts für das, was sonst Miscellanea oder Varia hieß, eine Rubrik für alles, was sich keiner Rubrik im sachlichen Sinn zuordnen ließ. Diese Rubrik der nicht-rubrifizierbaren Bücher war auch Sammelbecken für die Philosophie und für andere Werke außerhalb der $19 \mathrm{Ru}$ briken, vor allem aber war sie der Ort der Sammelbände - wohlgemerkt der eigens veranstalteten Sammelbände! Hundert Jahre später sah etwa der Katalog der Helmstedter Universitätsbibliothek für die Werke des Descartes die Rubrik "scriptores universae philosophiae" vor. Man nahm dann unproblematisch "Philosophie" selbst als Rubrik auf, und strukturierte die Sachkataloge nach deren Unterteilungen, analog den universitären Disziplinen innerhalb der Philosophischen Fakultät; so kam etwa auch der Philologie ein Platz zu. Im 17. Jahrhundert wurde Philosophie jedoch kaum als einzelne Wissenschaft aufgefasst, so dass eine Ordnung wie bei August, die eine dem Quadrivium und dem Trivium angelehnte Bildungsordnung war, keineswegs aus dem Rahmen fiel. Er hatte alle sieben freien Künste - Musik, Geometrie, Astronomie, Arithmetik und Rhetorik, Grammatik, Dialektik bzw. Logik - berücksichtigt.

Dass Descartes hauptsächlich bei den Quodlibetica landete, war also weniger eine intellektuelle Verlegenheit als eine praktische Besorgnis, denn das Zusammenbinden zeitgleich erworbener und formatgleicher Werke führte ganz oft dazu, den fertigen Band dorthin zu stellen, wo sein heterogener Inhalt nicht anstößig werden konnte: an den Rand der Rubriken. Dass bei August durchschnittlich vier Schriften in einem Band vereinigt wurden, zeigt einen gewiss außergewöhnlich hohen Anteil an Sammelbänden an. Grund für die Sammelbandmanie war, neben Sparsamkeitserwägungen, auch das hohe Tempo der Anschaffungen, das es bequem möglich machte, ohne Zeitverlust Texte zu kombinieren und der Sammlung einzuverleiben. Der Herzog beschäftigte mehrere Buchbinder gleichzeitig und hatte wohl immer genug für alle zu tun. ${ }^{29}$ So erklärt sich pragmatisch aus dem raschen Aufbau der Sammlung die effektive Zerstreunng von Werken, die Atomisierung von Autoren und die Offenheit des Rubrikenschemas, das nicht nur keine strikten Grenzen zwischen den Rubriken artikulierte, sondern auch keinerlei interne Gliederung. August katalogisierte nicht retrospektiv, sondern prospektiv, und das nicht nach philosophischer Struktur, sondern mit einem Interesse wohl vor allem an den einzelnen Texten. Was darüber hinaus an Zusammenhang der Bände ausgesagt wurde, war im Katalog realisiert.

\section{Verweisungen oder Der Ort der Bücher in der »memoria localis «}

Es gab noch einen weiteren Grund für die relative Großzügigkeit von August, was die Einhaltung der von ihm selbst skizzierten sachlichen Ordnung angeht: Er stattete seinen Katalog mit Verweisungen aus, die zusätzlich zu den Verweisungen im alphabetischen Index Beziehungen zwischen den Büchern nachzuzeichnen erlaubten und die wieder zusam-

27 Otegen: A Bibliography of the Works of Descartes, S. 18, $40,116,183,188,219,264,301,371,415,499,584,736$.

$28 \mathrm{Vgl}$. Ulrich Johanmes Schneider: Ordnung als Schema und als Operation. Die Bibliothek Herzog Augusts. In: Foucault und die Künste. Hrsg. von Peter Gente. Frankfurt am Main: Suhr* kamp 2004, S. 315-338.

29 Vgl. Nicholas Pickwoad: Bookbindings in the Bibliotheca Augusta. In: A Treasure House of Books, 5. 65-104. 


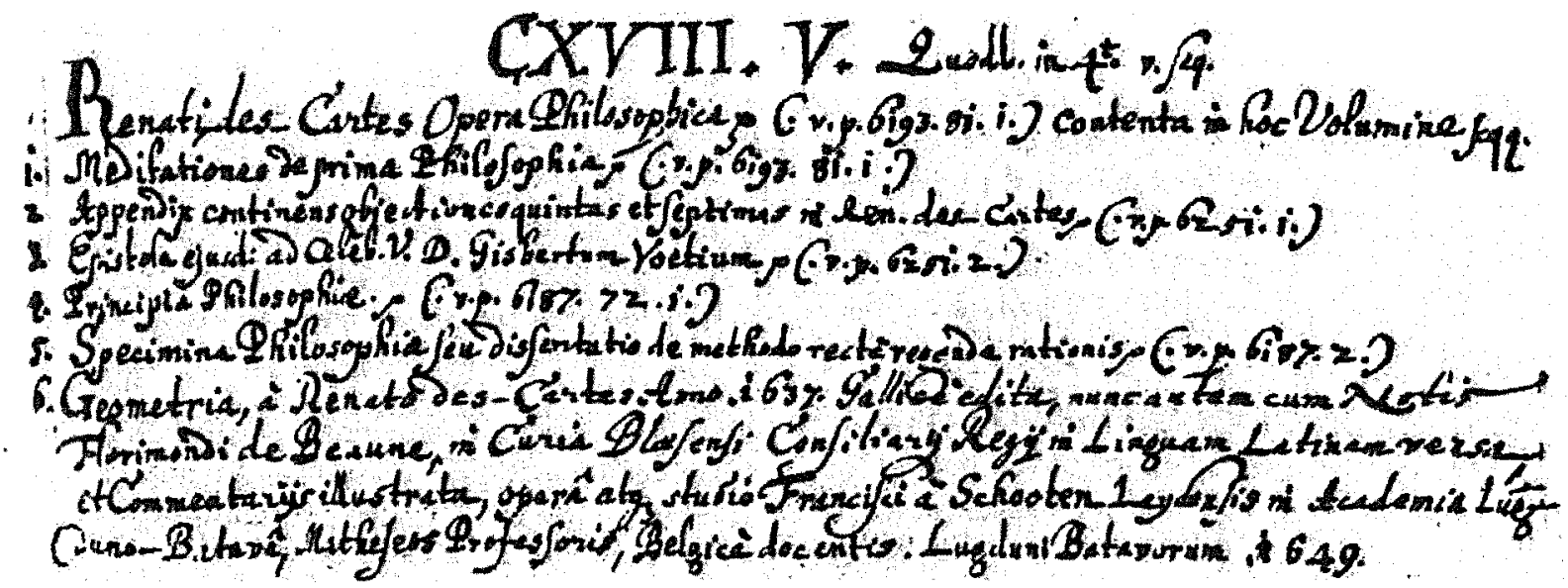

Abb. 8a: Verweisungen beim Eintrag Descartes im Bücherrad-Katalog (Opera philosophica) auf S. 6255 (Detail).

menzuführen halfen, was im Regal getrennt war. Um auf das Beispiel Descartes zurückzukommen: Es gab insgesamt 17 Stellen, an denen man Werke von Descartes im Regal antreffen konnte. Diese Stellen wurden im Katalog einmal aufgeführt, bei der Hauptaufnahme, worauf aber auch von anderen Seiten verwiesen wurde. Das geschah nicht systematisch und vollständig, aber oft. Diese Verweisungen standen am Rand des Katalogeintrags (etwa desjenigen einer anderen Schrift desselben Autors), wofür ein absichtlich breiter Rand vorgesehen war.

Gelegentlich wurden Verweisungen auch bei der Aufnahme selbst notiert, wie man bei der DescartesWerkausgabe von 1650 auf Seite 6255 erkennen kann. Meist aber war es ein eigener Durchgang, der zu den Verweisungen führte, also gewissermaßen eine Lektüre des Katalogs, anch wenn das schwierig zu denken ist. Derart wurde die multiple Existenz des Werkes von René Descartes im Bücherregal durch den Katalog wieder eingeschränkt oder konzentriert. Dabei gaben die Verweisungen innerhalb des Katalogs neben der Seitenzahl nur ausnahmsweise die Signatur komplett, meist nur einen eindeutigen Teil davon an, im Regelfall die Nummer vor dem entsprechenden Teil des Sammelbandes.

So wird auf der Seite 6255 von der Signatur Quod. 118.5 (Opera 1649) auf die Signatur Quod. 81.1 auf der Seite 6193 (Opera 1650) ausnahmsweise vollständig verwiesen (Abb. 8a), von dort aber am Rand anf die Signatur Theol. 163.23 auf der Seite 4245 wie meist üblich nur durch einen Punkt nach der Seitenzahl (Abb. 8b), zusammen mit der Ziffer 2, die besagt, dass man dann auf der entsprechenden Seite die Méditationes von 1644 als zweite beigebundene Schrift suchen muss. Auf der Ziel-Seite ist dann die Schrift leicht neben der
Ziffer 2 zu finden, leichter sogar als mit Angabe der vollen Signatur. Das System der Signaturen und der entsprechend angelegten Titelanzeigen wird vom Katalog wie ein Text behandelt, den er selber indiziert.

Ein anderes Beispiel für Verweisungen ist ein Buch von Simon Schard über die Prinzipien der Kaiser- und Kurfürstenwahl in Deutschland. Schard war Beisitzer im Reichskammergericht, er verstarb 1573, gelangte aber mit diesem Werk im 17. Jahrhundert zu neuen Ehren. Es findet sich in fünf Ausgaben auf den Seiten 729, 782, 1186, 2560 und 3500 verzeichnet, drei verschiedenen Rubriken zugeordnet: Politica, Historica, Quodlibetica. ${ }^{30}$ Von allen zwischen diesen Einträgen überhaupt möglichen sechzehn Verweisungen sind, wie im Fall der Werke von Descartes, nur wenige realisiert. Das Verweisungssystem des Hauptkatalogs ist selbst ein Produkt der sukzessiven Erweiterung, was sich darin ausdrückt, dass von neu eingetragenen Titeln öfter auf alte Eintragungen verwiesen wird als umgekehrt.

Man muss sich den Bibliothekar in Aktion vorstellen. Er arbeitet am eigens zum Katalogisieren gebauten Bücherrad (Abb. 9), das alle Bände des Katalogs aufnimmt und Platz für die Ablage weiterer Folianten bietet. Nach der Titelaufnahme wird am Hebel gezogen, die Pulte drehen sich umeinander, damit - mittels herzoglicher memoria und nach Konsultation der alphabetischen Indices - in den jeweils nach vorne beförderten Bänden einige Verweisungen eingetragen werden können. Der Katalog als

30 Simon Schard: De Principium, quibus Electio Imperatoris in Germania commendata est, origine, seu institutione liber unus. StraßBburg 1608: 487 Hist. $8^{\circ}, 43$ Quod. $2^{\circ}, 76.1$ Quod. $2^{\circ}$, 2 Pol. $2^{\circ}, 107.5$ Pol. $2^{\circ}$. 


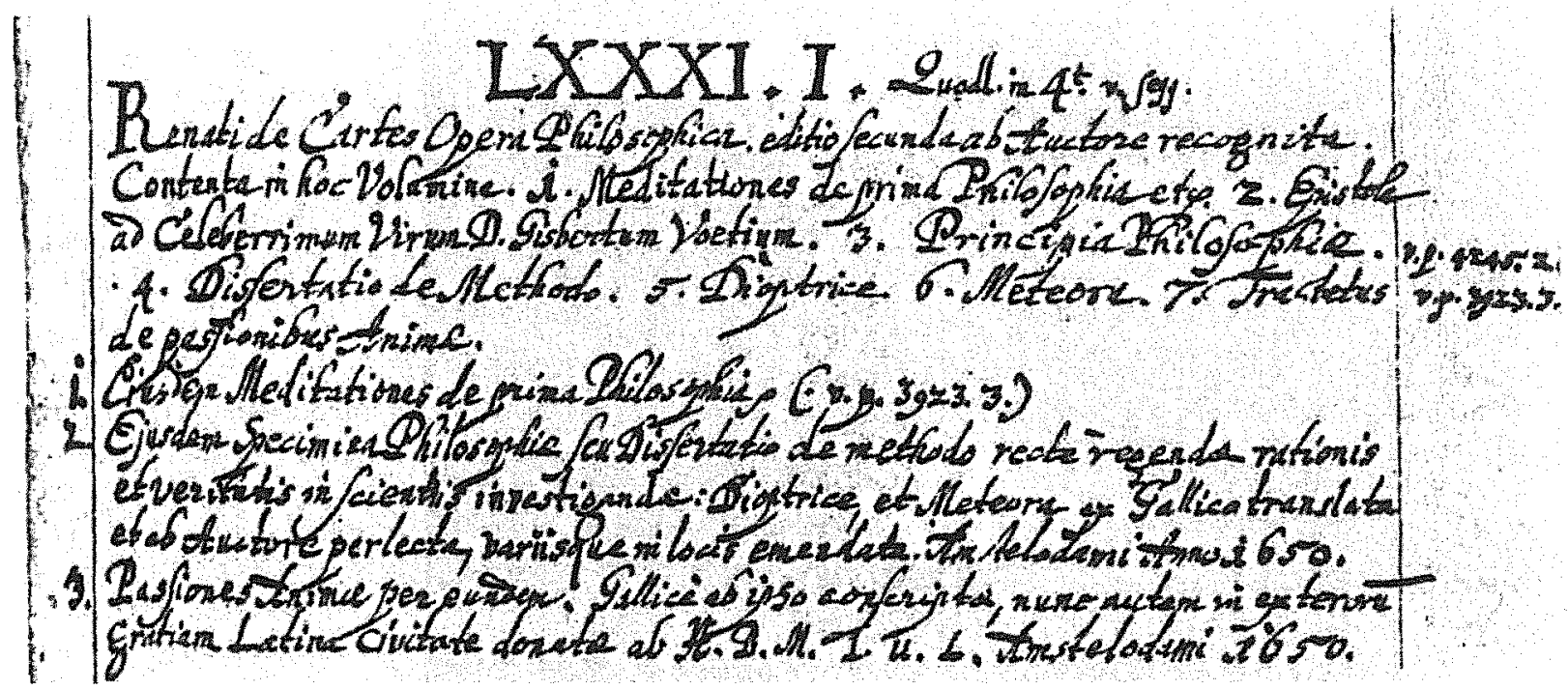

Abb. 8b: Verweisungen beim Eintrag Descartes im Bücherrad-Katalog (Opera philosophica) auf S. 6193 (Detail).

ein immer geöffnetes Manuskript wird auf den beweglichen Pulten zur Verweismaschine. ${ }^{31}$ Aber man sieltt auch leicht, wie das Rotieren der Katalogbände in ein eher hilfloses Kreisen ausarten kann und die vielfachen Bezugnahmen der Bücher nicht einzuholen vermag.

Kein Zweifel: Das System war genau, es wurde von späteren Schreibern und Benutzern bis über das Jahr 1700 hinaus weitergeführt. ${ }^{32}$ Aber so wenig die herzogliche Bibliothek eine strenge sachbezogene Buchaufstellung repräsentierte, so wenig war das System der Verweisungen dazu geeignet, die sachliche Kohärenz zu stärken. Allenfalls reparierte es die autor- und werkbezogenen Zerstreuungen. Der herzogliche Buchlokalisierungstrieb, den wir so gerne attestieren möchten, weil wir die Bibliothek vom allgemeinen Lesen und Benutzen her denken, war bei August beschränkt auf eine sehr knappe doppelstufige Indizierung vom alphabetischen Index auf den Hauptkatalog und innerhalb des Katalogs von bestimmten Titeln auf andere.

Wenn man einige Charakteristika dieses Systems der über fast ein ganzes Jahrhundert erfolgten Katalogisierung einer der größten Bibliotheken überhaupt hervorheben wollte, könnte man sagen, dass offenbar das Interesse an Wachstum und Bereicherung der Bestände das Interesse an sachlicher Gruppierung der Bücher übertraf. August war an schneller Zugänglichkeit interessiert, und dafür hielt er sein System für ausreichend.

Die Zugänglichkeit der Bücher stand wohl auch hinter der Tendenz zur Produktion von Sammelbänden. Der Herzog war seit seiner Studienzeit mit der Technik des Exzerpierens vertraut; er hatte lan- ge ein Buch geführt, in das er nach der Methode der loci communes Sentenzen verzeichnete, die ihm bei der Lektüre "merk-würdig erschienen. ${ }^{33}$ August. las sowieso im Zusammenhang und verstand Bücher als kontinuierliche Größen eines Universums an Wissen. (Mangels eigener Aussage seitens des Herzogs muss eine solche Beschreibung allerdings Rekonstruktion bleiben.) Mindestens aber kann man sagen, dass auch der Eintrag des Titels in den Katalog einen Lektüreakt repräsentierte, denn bei dieser Gelegenheit sind wohl auch Marginalien entstanden. Wie von Katte zeigte, hatte August mehrere in größerem zeitlichen Abstand gekaufte Exemplare ein und derselben Schrift von Luther mit Marginalien versehen, offenbar im Zusammenhang mit der Katalogaufnahme. ${ }^{34}$ Ein anderes Zeichen für den Lektürecharakter der Titelaufnahme sind nach-

31 Das Bücherrad soll August nach eigenen Plänen haben bauen lassen; vgl. Yorck-Alexander Haase: Die Geschichte der Herzog August Bibliothek in sechs Stationen dargestellt. In: Wolfenbütteler Beiträge 2 (1973), S. 17-42, S. 26.

32 Katte: Herzog August und die Kataloge seiner Bibliothek, weist S. 182 nach, dass die Einträge im Bücherradkatalog ca. 1698 aufhören und nur gelegentlich noch, allerdings mindestens bis 1719 , Nachträge gemacht wurden.

$33 \mathrm{Vgl}$. den Beitrag von Gilbert Heß: Fundamente fürstlicher Tugend. Zum Stellenwert der Sentenz im Rahmen der voruniver* sitären Ausbildung Herzog Augusts d. J. von Braunschweig-Lüneburg (1579-1666). In: Sammeln, Ordnen, Veranschaulichen: zur Wissenskompilatorik in der Frühen Neuzeit. Hrsg. von Frank Büttner, Markus Friedrich, Helmut Zedelmaier. Münster: Lit Verlag 2003, S. 131-173.

34 Katte: Herzog August und die Kataloge seiner Bibliothek, S. $188 \dot{f}$. 


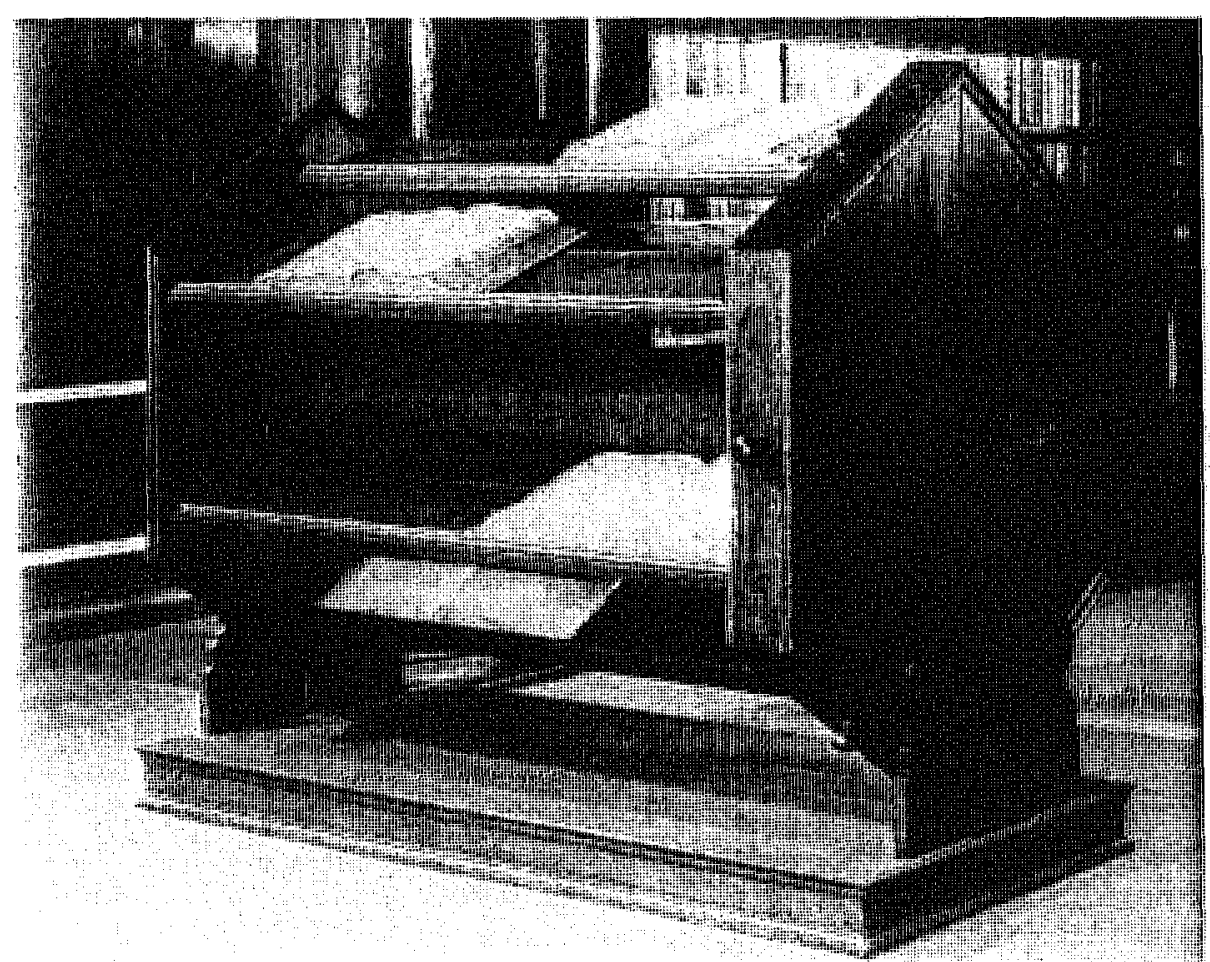

Ab6. 9: Das Bücherrad (ca. 1625): Instrument der Katalogisierung.

localis« im großen Kontinuum des gesammelten Wissens. Ein Katalog sei, wird der Göttinger Universitätsbibliothekar und Orientalist Johann David Michaelis später bemerken, ein Hilfsmittel zur Auffindung von Büchern für diejenigen, die keine solche "memoria localis" haben. ${ }^{36}$ Im Falle von August war das eine einsame Übung, noch kein >Benutzungsfalk.

August hat mit seiner Bücherordnung möglichst wenig in die schon mitgebrachte Ordnung der Bücher eingegriffen, vielmehr den komplexen Verweisungen aus den Büchern heraus seine eigene Verweisungsmaschine an die Seite gestellt. Die Wolfenbütteler Bib-

träglich eingefügte ausführliche Inhaltsangaben zu einzelnen Bänden. ${ }^{35}$

So lässt sich der sogenannte Katalog als Dokument einer Praxis verstehen, die begleitend zur Aufstellung der Bücher diese bis ins Einzelne protokolliert und sie wieder in ein Manuskript überführt, wo sie zusammenstehen: im Regal, wo die beschriebenen Rücken einander eng berühren, und im Kata$\log$, wo zusätzliche Verweisungen ein Netz von $\mathrm{Zu}$ sammenhängen entfalten, das allerdings ein privates Netz war, das Netz des privilegierten Lesers, den August selbst vorstellte. Vielmehr als Kataloge für irgendwelche oder gar anonyme Leser waren die "Handbücher", die August in seinem "Gemach" führte, Hilfsmittel der Buchfindung und -identifizierung allein für ihn selbst, Stütze der »memoria liothek blieb sozusagen durchsichtig auf die vielen möglichen Ordnungen hin, nicht definierend wie spätere Realkataloge zum Nutzen akademischer Ausbildung, sondern gewissermaßen rezipierend. Der Katalog als solcher, ohne Standortverzeichnis und alphabetisches Register, allein als Text betrachtet, war ein Manuskript der Unordnung. ${ }^{37} \mathrm{Er}$ war aber eben kein Buch, und das heißt: Er war nicht nur keine abgeschlossene bibliographische Einheit, er stand nicht nur nicht in den Regalen und war auch nicht - wie viele Kataloge nach ihm - selbst in einem Katalog verzeichnet, sondern eher ein Kaufregister, ein Lesetagebuch, ein Gedächtnisprotokoll und ein Itinerar durch eine nach Größe und Sorgfalt einmalige Büchersammlung des 17. Jahrhunderts.

35 So beginnen beispielsweise Inhaltsangaben zu Luthers Werken, die auf S. 45 eingetragen sind, auf der etwa vier Jahre später angelegten S. 2149.

36 Johann David Michaelis: Lebensbeschreibung vom ihm selbst abgefaßt. Leipzig: Barth 1793, S. 87.

37 Zur "Entkoppelung von Sachkatalog und Buchaufstellung" als Lösung für dieses Problem vgl. Uwe Jochum: Kleine Bibliotheksgeschichte. 2. Aufl. Stuttgart: Reclam 1999, S. 136. 


\title{
ARCHIV FÜR GESCHICHTE DES BUCHWESENS
}

\author{
Im Auftrag der \\ Historischen Kommission \\ des Börsenvereins des Deutschen Buchhandels e.V.
}

\author{
Herausgegeben von \\ Monika Estermann, Ursula RautenberG \\ UND REINHARD WITTMANN
}

Band 59

K·G·Saur München 2005 


\section{INHALT}

Ursula RAUTENBERG: Vorwort. Buch und Bibliothek als Wissensräume in der Frühen Neuzeit

IX

\section{TAGUNGSBEITRÄGE}

MARGareT M. SMITH: From Manuscript to Print: Early Design Changes .......................................... 1

OLIVER DUNTZE: Text und Kommentar in juristischen Drucken der Frühen Neuzeit .......................... 11

RENaTE WITTERN: Die Präsentation des anatomischen Wissens im Buch des 16. Jahrhunderts ............. 34

ARNDT BRENDECKE: 'Durchschossene Exemplare، Über eine Schnittstelle

zwischen Handschrift und Druck 50

MONIKA EstermanN: Signete und Widmungsbriefe Frankfurter Verleger

des späten 16. Jahrhunderts

UlRICH JOHANNES SCHNEIDER: Der Ort der Bücher in der Bibliothek und in Katalog

am Beispiel von Herzog Augusts Wolfenbütteler Büchersammlung

AlFredo SERraI: Bibliothekarische Kataloge als Spiegel und Instrumente von Wissensordnungen in der Frühen Neuzeit

\section{BEITRÄGE}

HANS-OTTO KEUNECKE: Bibelverlag und Bibeldruck im Fürstentum Ansbach

NADINE LANDECK: Gestaltungsraster in der Buchtypographie.

Die Entwicklung des typographischen Rasters vom Bauhaus bis zur Schweizer Grafik

und seine formalen und funktionalen Eigenschaften

Register

VOLKER TITEL: Electronic Publishing und E-Commerce im Buchhandel.

Ein Forschungsbericht für den Publikationszeitraum 1995 bis 2004

Register

\section{REZENSIONEN}

Evgenij L. Nemirovskij: Gutenberg und der älteste Buchdruck in Selbstzeugnissen.

(Bettina Wagner) 236

Internationale Bibliographie zur Papiergeschichte (IBP). (Peter F. Tschudin)

La vita nei libri: edizioni illustrate a stampa del Quattro e Cinquecento dalla Fondazione Giorgio Cini. FRANCESCO NOVATI: Scritti sull'editoria popolare nell'Italia di antico regime. (Luisa Rubini)

Bandregister

Anschriften der Herausgeber und Verfasser 